Check for updates

Cite this: Phys. Chem. Chem. Phys., 2017, 19, 24678

Received 20th May 2017, Accepted 7th August 2017

DOI: $10.1039 / c 7 c p 03395 h$

rsc.li/pccp

\title{
Orientational ordering of water in extended hydration shells of cations is ion-specific and is correlated directly with viscosity and hydration free energy
}

\author{
Yixing Chen, (D) Halil I. Okur, (D) Chungwen Liang (D) and Sylvie Roke (DD*
}

\begin{abstract}
Specific ion effects in aqueous solutions are investigated at the molecular, nanoscopic and macroscopic levels. Femtosecond elastic second harmonic scattering (fs-ESHS) is used here to assess the chemical effects of ions on molecular and nanoscopic length scales of water, probing changes in the charge distribution around ions as well as structural orientational order of water molecules in extended hydration shells. We measured $>0.05 \mathrm{M}$ electrolyte solutions with a series of chloride salts $(\mathrm{LiCl}, \mathrm{NaCl}$, $\mathrm{KCl}, \mathrm{CsCl}, \mathrm{RbCl}, \mathrm{NH}_{4} \mathrm{Cl}, \mathrm{MgCl}_{2}, \mathrm{CaCl}_{2}$, and $\mathrm{SrCl}_{2}$ ). Ion specificity is observed in both the local electronic anisotropy and the nanoscopic orientational ordering of water. Both observables are influenced more by cations with larger valencies and smaller sizes and follow a direct Hofmeister trend. These ion-induced structural changes in the hydrogen-bond network of water are strongly correlated with the viscosity $B$-coefficient and the Gibbs free energy of hydration of ions. Such a connection between the nanoscopic and macroscopic changes provides a possibility to construct a molecular model for specific ion effects in aqueous solutions.
\end{abstract}

\section{Introduction}

Inorganic ions play specific and irreplaceable roles in various physical, chemical, and biological processes in aqueous solutions, from colloid assembly and bubble coalescence to protein folding and enzyme activities, typically observed at and above the physiological concentration of electrolytes $(\sim 0.1 \mathrm{M}) .{ }^{1-6}$ Specific ion effects are shown to be present in various macroscopic properties of bulk aqueous solutions, such as viscosity of electrolyte solutions and solvation free energy of ions. ${ }^{7-9}$ Recent work also shows that changes occur at the molecular level. ${ }^{10-12}$ Neutron and X-ray diffraction studies that measure the structure factor of ionic hydration shells report ion-specific coordination numbers of alkali ions. ${ }^{13-16}$ The electronic state of ion hydrating water molecules was probed by X-ray absorption spectroscopy. It was found that cations with a low charge density weaken the hydrogen $(\mathrm{H})$-bonds of hydrating water molecules whereas cations with a high charge density cause the formation of strong H-bonds. ${ }^{17,18}$ Raman spectroscopy, ${ }^{19,20}$ vibrational dynamics measurements, ${ }^{21-24}$ and ensemble infrared photodissociation spectroscopy ${ }^{25,26}$ probe the $\mathrm{O}-\mathrm{H}$ stretch modes of hydrating water molecules in

Laboratory for fundamental BioPhotonics (LBP), Institute of Bio-engineering (IBI), and Institute of Materials Science (IMX), School of Engineering (STI), and Lausanne Centre for Ultrafast Science (LACUS), École Polytechnique Fédérale de Lausanne (EPFL), CH-1015, Lausanne, Switzerland. E-mail: sylvie.roke@epfl.ch the vicinity of ions. These studies showed that different ions perturb the water network and restrain the dynamics of water molecules to different extents, leading to ion-specific changes in the spectra of $\mathrm{O}-\mathrm{H}$ stretch regions. Changes in the complex permittivity of electrolyte solutions as probed by dielectric spectroscopy measurements indicated that small and highly charged cations align and irrotationally bind water in the first hydration shell. ${ }^{27}$ Auger electron spectroscopy studies of ultrafast charge delocalization phenomena in electrolyte solutions showed that cations with larger charges induce a slower electron-hopping rate to the oxygen of hydrating water molecules. ${ }^{28,29}$ Computer simulations that calculated the radial distribution and orientation of water molecules in the ionic hydration shells ${ }^{4,19,23,30-33}$ showed that ions induce distinct changes in the water network that involve changing the number of H-bonds per water molecule and affecting the reorientation of individual water molecules.

All the above studies report specific ion effects on the structure and dynamics of water molecules in the first few hydration shells of ions in aqueous solutions. However, the mechanism of specific (cat)ion effects is not fully understood. ${ }^{34}$ A well-known example is the high selectivity of ion channels for either $\mathrm{K}^{+}$or $\mathrm{Na}^{+}$ions, which cannot be explained simply by a difference in geometry alone. ${ }^{35,36}$ Another example is that $\mathrm{Na}^{+}$ is essential to regulate blood pressure but toxic at a high intracellular concentration, whereas $\mathrm{K}^{+}$is not toxic and serves 
as the major intracellular monovalent ion. ${ }^{37}$ Although some recent molecular dynamics studies ${ }^{38-41}$ have reported ion-induced water ordering over nanoscale distances, there is no molecularlevel experimental evidence for long-range specific ion effects on the bulk water structure. An overarching molecular model based on combined experimental data and theory that explains how specific ion effects extend from the first few hydration shells of ions to the bulk water network and lead to macroscopic effects in the bulk properties of electrolyte solutions is still lacking. To help with this issue, a technique that is able to probe hydrating water at the molecular level and is also sensitive to longer range structural changes in extended hydration shells is needed. Femtosecond elastic second harmonic scattering (fs-ESHS) has recently been shown to access molecular level properties of electrolyte solutions up to nanoscale distances. ${ }^{39,42}$ Specifically, fs-ESHS studies on electrolyte solutions with ionic strengths in the range of $1 \mu \mathrm{M}$ to $100 \mathrm{mM}$ found a universal long-range $(\sim 20 \mathrm{~nm})$ influence of ions on the H-bond network of water molecules, which can be qualitatively explained as a perturbation in the water-water correlations induced by the total electrostatic field of the ions in solution. $^{39,43}$ As a second-order nonlinear process, fs-ESHS selectively probes both local electronic anisotropy of individual molecules and nanoscopic structural anisotropy (i.e. orientational order) in a liquid. Electronic anisotropy gives rise to an incoherent response and structural anisotropy gives rise to a coherent response that reflects the orientational order of molecules, whereas no second harmonic ( $\mathrm{SH}$ ) intensity is generated in a centrosymmetric environment composed of centrosymmetric molecules. The coherent and incoherent contributions can be investigated separately using different polarization combinations of the incoming and outgoing electromagnetic waves. The sensitivity to the orientational order as well as the electronic charge distribution suggests that fs-ESHS could also provide molecular level insight into specific ion effects in more concentrated aqueous solutions. As the orientational order in the H-bond network is related directly to the formation and breaking of $\mathrm{H}$-bonds, a connection with macroscopic properties of electrolyte solutions, such as viscosity and the Gibbs free energy of hydration, is worth investigating.

Here, we probe aqueous solutions, with ionic strengths $>0.05 \mathrm{M}$, of a series of chloride salts ( $\mathrm{LiCl}, \mathrm{NaCl}, \mathrm{KCl}, \mathrm{CsCl}$, $\mathrm{RbCl}, \mathrm{NH}_{4} \mathrm{Cl}, \mathrm{MgCl}_{2}, \mathrm{CaCl}_{2}$, and $\mathrm{SrCl}_{2}$ ) with fs-ESHS. We observe ion specificity in both the local electronic anisotropy and the nanoscopic orientational ordering of water in extended hydration shells. Both observables are influenced more by cations with larger valencies and smaller sizes and follow a direct Hofmeister trend. We also find that the ion-induced orientational ordering in the water network, revealed by a linear increase in the fs-ESHS intensity of the coherent response as a function of the electrolyte concentration, is strongly correlated with the viscosity $B$-coefficient and the Gibbs free energy of hydration of ions. These findings are of particular interest, given that cations play a critical role in biologically relevant aqueous systems. The observed connection between the molecular (electronic anisotropy), nanoscopic (orientational ordering of water) and macroscopic (viscosity/hydration free energy) observables will further our understanding of the hydration of ions as well as specific ion effects in aqueous solutions and will open a route to the development of a multiscale model.

The present work is structured as follows: we first introduce the theoretical background of fs-ESHS, followed by the Materials and methods section. Then we present theoretical calculations of the SH intensity of aqueous solutions of a series of chloride salts. The incoherent $\mathrm{SH}$ responses from single spherical ions with different radii and single water molecules are considered first. The coherent $\mathrm{SH}$ intensity is calculated for electrolyte solutions based on Debye-Hückel (DH) theory, in which a dielectric continuum, a gas of water dipoles and statistical distributions of ions that follow from the linearized Boltzmann distribution are assumed. We compare these relatively simplistic theoretical results with the data of fs-ESHS measurements of electrolyte solutions for the incoherent and coherent responses separately. We find that the experiments contain many details about the intricate relationship of water and ions that are not captured by the models. Finally, we correlate the fs-ESHS responses obtained in different polarization combinations with the viscosity $B$-coefficient and Gibbs free energy change of hydration to show a connection between the nanoscopic and macroscopic observations. Such a connection evidently warrants further investigation into the atomistic details that underlie the presented experiments.

\section{Theoretical background of fs-ESHS}

In a fs-ESHS process in an aqueous electrolyte solution, $\mathrm{SH}$ polarization $\boldsymbol{P}^{(2)}(2 \omega)$ is induced in the focal volume of incident electromagnetic waves $\boldsymbol{E}(\omega)$ with frequency $\omega$ and wavevector $\boldsymbol{k}_{\omega}$. $\boldsymbol{P}^{(2)}(2 \omega)$ is the source of SH light with frequency $2 \omega$ and wavevector $\boldsymbol{k}_{2 \omega}$. The $\mathrm{SH}$ response of a molecule is characterized by its second-order hyperpolarizability, $\boldsymbol{\beta}^{(2)}(2 \omega ; \omega, \omega)=\boldsymbol{\beta}^{(2)}$. When an electrostatic field $\boldsymbol{E}(0)$ is present, the third-order hyperpolarizability $\boldsymbol{\beta}^{(3)}(2 \omega ; \omega, \omega, 0)=\boldsymbol{\beta}^{(3)}$ will also contribute to the emitted SH light. The emitted fs-ESHS intensity $I(2 \omega)_{J}$ measured at a scattering angle $\theta=90^{\circ}$ (Fig. 1A) in a polarization state $J$ is: ${ }^{44,45}$

$$
\begin{aligned}
& I(2 \omega)_{J} \propto\left|P^{(2)}(2 \omega)_{J}\right|^{2}=E(\omega)_{L}{ }^{4}\left|\sum_{h=1}^{N} \bar{\beta}_{J K L}(h) \mathrm{e}^{i \boldsymbol{q} \cdot \boldsymbol{r}_{h}}\right|^{2}, \\
& \bar{\beta}_{J K L}(h)=\frac{1}{2} \beta_{J K L}^{(2)}(h)+\frac{1}{6} \beta_{J K L M}^{(3)}(h) E(0)_{M},
\end{aligned}
$$

where $\boldsymbol{r}_{h}$ is the position of the $h$-th molecule. $\sum_{h=1}^{N}$ represents the sum over molecules in a unit volume of the focus of the incident light. The indices $J, K, L$, and $M$ denote the polarization states of the involved electromagnetic fields. They are ordered from high (left) to low frequency (right). The scattering vector $\boldsymbol{q}$ is defined as $\boldsymbol{q} \equiv \boldsymbol{k}_{2 \omega}-2 \boldsymbol{k}_{\omega}$. The fs-ESHS intensity $I(2 \omega)$ can be considered as arising from a self-correlation term that is incoherent $\left(\left\langle\bar{\beta}_{J K L}{ }^{2}\right\rangle_{\text {incoh }}\right)$ and a 


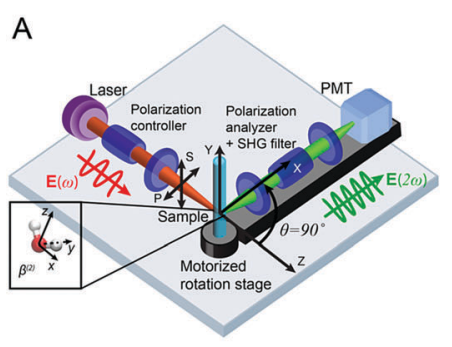

Incoherent response

Coherent response
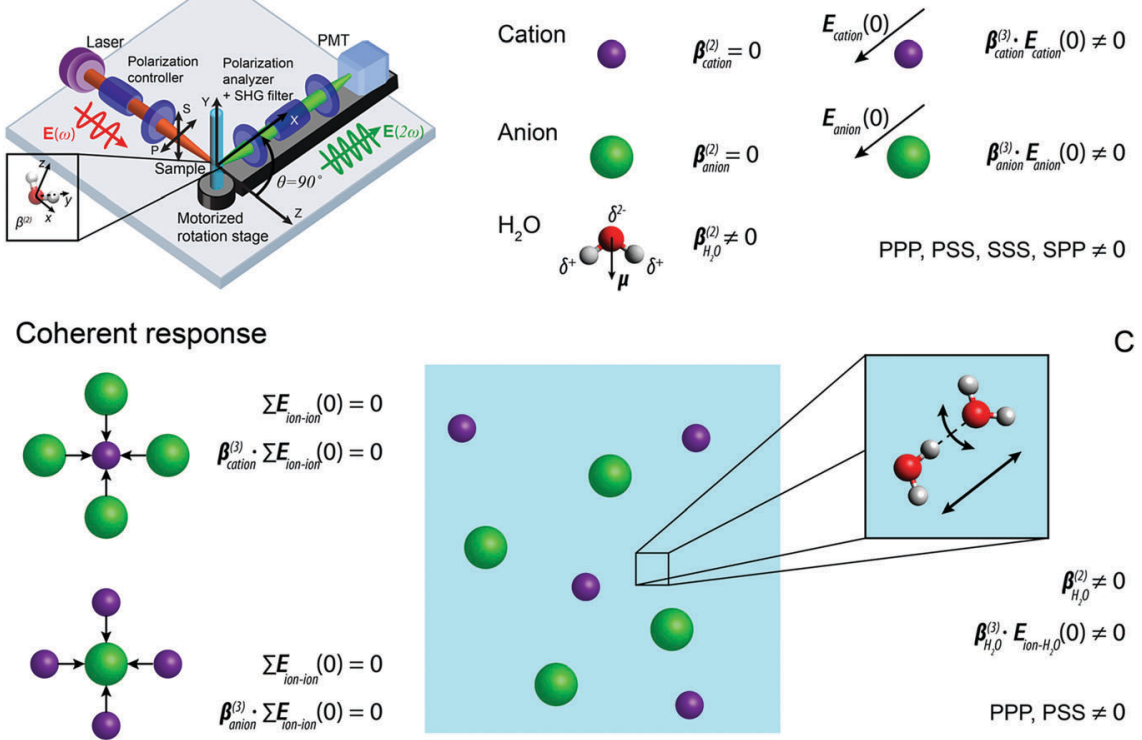

PPP, PSS, SSS, SPP $\neq 0$

Fig. 1 (A) Sketch of the fs-ESHS experiment and the different coordinate systems. $P(S)$ represents linear polarized light parallel (perpendicular) to the scattering plane (i.e., the XZ plane). (B) Illustration of possible incoherent contributions to the fs-ESHS responses of spherical ions (row 1 and 2) and water molecules (row 3) in an electrolyte solution. The centrosymmetric ions have a vanishing second-order hyperpolarizability $\left(\boldsymbol{\beta}^{(2)}=0\right)$, while the non-centrosymmetric water molecules have a non-zero hyperpolarizability. When an electrostatic field is present, the centrosymmetry around the ion is broken and SH photons can emerge. The non-zero polarization combinations in a collinear excitation geometry are: PPP, PSS, SSS, and SPP. This three-letter code for the polarization state, from left to right, denotes the polarization state of each participating photon from high frequency to low frequency. (C) Illustration of possible coherent contributions to the fs-ESHS responses of ions (left) and water molecules (right). For the ion response the electrostatic field from other ions is on average vanishing, leading to a vanishing coherent contribution as summarized by the equations $\left(E_{\text {ion-ion }}(0)\right.$ represents the electrostatic field from ions on a central ion). For the water response, the second-order hyperpolarizability of water is non-zero, as is the electrostatic field from the surroundings, ensuring that both are sources of coherent $\mathrm{SH}$ light, as summarized by the right equations $\left(E_{\mathrm{ion}-\mathrm{H}_{2} \mathrm{O}}(\mathrm{O})\right.$ represents the electrostatic field from ions on water molecules). These responses correspond to changes in the structural orientational correlations in the solution and can only be probed in the PPP and PSS polarization combinations. The inset illustrates two correlated water molecules connected by a $\mathrm{H}$-bond. The black arrows represent two directions along which the $\mathrm{H}$-bond can be broken (via bending or stretching). Within this decomposition, fs-ESHS is only sensitive to the bending motion of the $\mathrm{H}$-bond. ${ }^{39}$

cross-correlation term that is coherent $\left(\left\langle\bar{\beta}_{J K L}{ }^{2}\right\rangle_{\text {coh }}\right)$, which changes eqn (1) to ${ }^{44}$

$$
\begin{aligned}
I(2 \omega)_{J} \propto & E(\omega)_{K}^{2} E(\omega)_{L}^{2} \\
& \times\left\{\sum_{h=1}^{N}\left|\bar{\beta}_{J K L}(h)\right|^{2}+\sum_{h \neq h^{\prime}} \bar{\beta}_{J K L}(h) \bar{\beta}_{J K L}\left(h^{\prime}\right) \mathrm{e}^{i \boldsymbol{q} \cdot\left(\boldsymbol{r}_{h}-\boldsymbol{r}_{h^{\prime}}\right)}\right\} \\
= & E(\omega)_{K}^{2} E(\omega)_{L}^{2} N_{\mathrm{m}}\left\{\left\langle\bar{\beta}_{J K L}{ }^{2}\right\rangle_{\text {incoh }}+\left\langle\bar{\beta}_{J K L}{ }^{2}\right\rangle_{\mathrm{coh}}\right\},
\end{aligned}
$$

where $N_{\mathrm{m}}$ is the number density of molecules in the focal volume. \langle\rangle represents an ensemble average with respect to time and space. $\mathrm{e}^{i \boldsymbol{q}\left(\boldsymbol{r}_{h}-\boldsymbol{r}_{h^{\prime}}\right)}$ in the coherent term represents the orientational correlation between molecules $h$ and $h^{\prime}$. In a collinear fs-ESHS experiment, the two incident optical fields are identical $(K=L)$. The incoherent response reports on molecular electronic anisotropy. The coherent response reports on structural orientational correlations or orientational order between molecules. ${ }^{44}$

\section{Materials and methods}

\section{Sample preparation}

$\mathrm{MgCl}_{2}$ (99.99\%, Sigma-Aldrich), $\mathrm{CaCl}_{2}$ (99.99\%, Sigma-Aldrich), $\mathrm{SrCl}_{2}$ (99.99\%, Sigma-Aldrich), LiCl (99.999\%, Sigma-Aldrich),
$\mathrm{NaCl}$ (99.999\%, Acros), $\mathrm{NH}_{4} \mathrm{Cl}$ (99.9995\%, Fluka), $\mathrm{RbCl}$ (99.95\%, Sigma-Aldrich), CsCl (99.9995\%, Fluka), and KCl (99.999\%, Acros) were used as received without further purification. All samples were prepared by dissolving the electrolytes in degassed ultra-pure water $(18.2 \mathrm{M} \Omega \mathrm{cm}$, Millipore, Inc.) to obtain a $5 \mathrm{ml}$ stock solution at a high concentration by using a $5 \pm 0.025 \mathrm{ml}$ volumetric flask (Duran) at room temperature. The stock solutions were filtered $(0.1 \mu \mathrm{m}$ PVDF membrane filters, Millex-VV, Millipore) and then diluted to the desired concentration. The relative error in the reported electrolyte concentrations is $0.5 \%$.

\section{Femtosecond elastic second harmonic scattering (fs-ESHS)}

$190 \mathrm{fs}$ laser pulses centered at $1028 \mathrm{~nm}$ with a repetition rate of $200 \mathrm{kHz}$ were used to probe the samples. The input pulses were linearly polarized using a Glan-Taylor polarizer (GT10-B, Thorlabs) and a zero-order half-wave plate (WPH05M-1030), and were cleaned using a longpass filter (FEL0750, Thorlabs). The cleaned laser pulses were then focused into a cylindrical glass sample cell (4.2 $\mathrm{mm}$ inner diameter) using a plano-convex lens $(f=75 \mathrm{~mm}$, LA1608-B, Thorlabs). The focused laser pulses had a beam waist of $\sim 35 \mu \mathrm{m}$ diameter and a Rayleigh length of $\sim 0.94 \mathrm{~mm}$. The pulse energy at the sample was $0.3 \mu \mathrm{J}$ (incident laser power $P=60 \mathrm{~mW}$ ). The generated fs-ESHS light was filtered using a bandpass filter 
(ET525/50m, Chroma) and was collected at a scattering angle of $90^{\circ}$ using a gated photomultiplier tube (PMT, H7421-40, Hamamatsu). The polarization of the collected fs-ESHS light was analyzed using a Glan-Taylor polarizer (GT10-A, Thorlabs). The acceptance angle for collecting the fs-ESHS light was set to $11.4^{\circ}$ and each data point was acquired with an acquisition time of $50 \times 1 \mathrm{~s}\left(50 \times 2 \times 10^{5}\right.$ pulses $)$ and a gate width of $10 \mathrm{~ns}$. The fs-ESHS intensity of pure $\mathrm{H}_{2} \mathrm{O}$ was measured between every two samples and was used to monitor and correct for laser fluctuations. All liquid samples were stored and measured in sealed glass sample cells at room temperature $(T=297 \mathrm{~K})$. The reproducibility of fs-ESHS measurements of electrolyte solutions was $1-3 \%$. More details of the fs-ESHS experiment system can be found in ref. 39 and 46 .

\section{Fits to the fs-ESHS data}

The fs-ESHS data were fitted with a linear function:

$$
y=k x+b
$$

where $x$ and $y$ are fitted to the relative intensity and concentration, $k$ is the fitted slope, and $b$ is the intersect of the $y$-axis of the linear curve. Linear fittings with this given equation were performed using the built-in fitting function "Line" in IGOR Pro 6 (WaveMetrics), in which the singular value decomposition algorithm ${ }^{47}$ is employed for this non-iterative curve fitting. The coefficient of determination $R^{2}$, indicating the quality of the linear fit, can be calculated by ${ }^{48}$

$$
R^{2} \equiv 1-\frac{\sum_{i}\left(y_{i}-f_{i}\right)^{2}}{\sum_{i}\left(y_{i}-\bar{y}\right)^{2}}
$$

where $y_{i}$ is the data value and $\bar{y}$ is the mean value of $y_{i}$, and $f_{i}$ is the corresponding predicted value. A value of $R^{2}$ closer to 1 is indicative of a better fit. The error bar in $k$ is taken to be determined by the $95 \%$ confidence interval.

\section{Molecular dynamics simulations}

Polarizable molecular dynamics (MD) simulations were performed using the TINKER 7.1 package ${ }^{49}$ to calculate the electrostatic field $\boldsymbol{E}(0)$ on an ion. The Amoeba14 model $^{50}$ was chosen for the water molecules, and the Amoeba ion parameters ${ }^{51}$ for the sodium and chloride ions. Cubic simulation boxes, with a side of $5 \mathrm{~nm}$, were prepared by combining $\sim 4000$ water molecules with randomly distributed pairs of ions, up to the concentration of $2 \mathrm{M}$. After initial equilibration of $100 \mathrm{ps}$, simulations were run for $500 \mathrm{ps}$, and snapshots of the instantaneous configurations of water and ions were saved for every $100 \mathrm{fs}$. During equilibration in the NPT ensemble, the velocity Verlet method was used with 1.0 fs integration time steps. The system temperature was kept at $298 \mathrm{~K}$ using an Andersen thermostat with a coupling time of $0.1 \mathrm{ps}$. The system pressure was kept at 1.0 atm using a Berendsen barostat with a coupling time of 2.0 ps. The particle mesh Ewald method ${ }^{52}$ was used to evaluate the long-range electrostatic interaction, and the real space cutoff for Ewald summation as well as for van der Waals interactions was set to 12.0 angstroms. The convergence threshold of $10^{-5} \mathrm{D}$ was applied during calculation of self-consistent induced dipoles. During production runs in the NVT ensemble, Langevin dynamics with a velocity Verlet integrator was employed. The Langevin dynamics used a friction coefficient of $1.0 \mathrm{ps}^{-1}$. The system pressure was kept at 1.0 atm using the Nosé-Hoover thermostat $^{53}$ with a coupling time of $0.1 \mathrm{ps}$.

\section{Results and discussion}

\section{A. Theory: expected fs-ESHS response from electrolyte solutions}

For an ionic solution, incoherent $\mathrm{SH}$ intensity from electronic anisotropy within individual water molecules or ions may be expected. We can also consider whether there are coherent contributions from structural orientational order between water molecules or ions to the fs-ESHS response. Both contributions are illustrated in Fig. 1B and $\mathrm{C}$ and will be considered below. In what follows we will first calculate the incoherent SH intensity from individual ions by using the results from a MD simulation in combination with a simple scaling law for $\boldsymbol{\beta}^{(3)}$ (which is used for describing non-resonant nonlinear optical interactions). ${ }^{54}$ Single spherical ions with different radii will be considered. The incoherent $\mathrm{SH}$ intensity from water molecules will be discussed briefly as well. Then, we will focus on the expected coherent $\mathrm{SH}$ intensity from water molecules as induced by ions. To estimate this coherent $\mathrm{SH}$ intensity from electrolyte solutions we make use of a mean field DH model. ${ }^{39}$ In this DH model, the medium is considered as a dielectric continuum. It is also assumed that the statistical distribution of ions follows the linearized Boltzmann distribution. Water molecules are considered as a non-interacting gas of dipoles.

Incoherent response: local electronic anisotropy. As shown in Fig. 1B, for centrosymmetric ions, the absence of molecular electronic anisotropy results in a vanishing $\boldsymbol{\beta}^{(2)}$ tensor $\left(\boldsymbol{\beta}_{\text {cation/anion }}^{(2)}=0\right)$. Spherical ions will therefore contribute primarily through the third-order response $\boldsymbol{\beta}^{(3)}$ that requires an interaction with two optical photons and an electrostatic field component $\left(\boldsymbol{\beta}_{\text {cation/anion }}^{(3)} \cdot \boldsymbol{E}_{\text {cation/anion }}(0) \neq 0\right)$. The electrostatic field originates from the surrounding water molecules and ions in the solution. A value for this electrostatic field $\boldsymbol{E}(0)$ is obtained from atomistic polarizable MD simulations using Amoeba models. ${ }^{50,51}$ These computations show that the electrostatic field $\boldsymbol{E}(0)$ that is felt by the ions originates primarily from the surrounding water molecules. We find concentration independent values of $\left|\boldsymbol{E}_{\text {anion }}(0)\right| \sim 12.2 \pm 4.2 \times 10^{9} \mathrm{~V} \mathrm{~m}^{-1}$ around the anions and $\left|\boldsymbol{E}_{\text {cation }}(0)\right| \sim 4.9 \pm 2.0 \times 10^{9} \mathrm{~V} \mathrm{~m}^{-1}$ around the cations. The contribution of other ions to $\boldsymbol{E}(0)$ is found to be negligible compared to that of neighboring water molecules, and the MD simulations of three different concentrations (13 mM, $40 \mathrm{mM}$, and $2 \mathrm{M}$ ) produced the same electrostatic field. Probably this is due to an on-average symmetric arrangement of ions around the central one as illustrated in Fig. 1C. In addition, these surrounding ions are further away than the surrounding water molecules. 
The average magnitude of $\boldsymbol{\beta}^{(3)}$ of the chloride ion was calculated previously using quantum chemistry calculations: ${ }^{55}$ $\left|\overline{\boldsymbol{\beta}}_{\mathrm{Cl}^{-}}^{(3)}\right| \sim 1.86 \times 10^{-60} \mathrm{C}^{4} \mathrm{~m}^{4} \mathrm{~J}^{-3}$. Knowing this value, we can compute $\boldsymbol{\beta}^{(3)}$ for other spherical ions by using the following scaling law for non-resonant nonlinear optical processes: ${ }^{54}$

$$
\left|\overline{\boldsymbol{\beta}}_{\text {ion }}^{(3)}\right|=\left|\overline{\boldsymbol{\beta}}_{\mathrm{Cl}^{-}}^{(3)}\right|\left(\frac{R_{\mathrm{ion}}}{R_{\mathrm{Cl}^{-}}}\right)^{7},
$$

where $R_{\text {ion }}$ is the ionic radius. The values used for the ionic radii are taken from the literature ${ }^{56,57}$ and listed in Table 1 . The incoherent fs-ESHS intensity from individual ions in water of an $\mathrm{AB}_{j}$ electrolyte is thus given by

$$
I_{\text {ion }}(2 \omega) \propto c\left\{\left(\left|\overline{\boldsymbol{\beta}}_{\text {cation }}^{(3)}\right|\left|\boldsymbol{E}_{\text {cation }}(0)\right|\right)^{2}+j\left(\left|\overline{\boldsymbol{\beta}}_{\text {anion }}^{(3)}\right|\left|\boldsymbol{E}_{\text {anion }}(0)\right|\right)^{2}\right\},
$$

where $c$ is the concentration of electrolytes in $M . j=1$ for chloride salts with monovalent cations and $j=2$ for divalent cations.

In contrast to spherical ions, the molecular hyperpolarizability is nonzero for dipolar water molecules $\left(\boldsymbol{\beta}_{\mathrm{H}_{2} \mathrm{O}}^{(2)} \neq 0\right)$, which thus gives rise to the leading-order contribution to the incoherent $\mathrm{SH}$ emission. Values of $\boldsymbol{\beta}^{(2)}$ of liquid water were obtained from an $a b$ initio study by Gubskaya and Kusalik. ${ }^{58}$ The incoherent response is expected to contribute to all four accessible polarization states (PPP, PSS, SPP, and SSS). The three-letter code for the polarization state, from left to right, denotes the polarization state of each participating photon from high frequency to low frequency. $P(S)$ represents the polarization direction of light parallel (perpendicular) to the $X Z$ horizontal scattering plane in Fig. 1A.

Coherent response: orientational order of water. In addition to an incoherent contribution to the fs-ESHS intensity, there is also a coherent contribution to $I(2 \omega)$ that reports on orientational correlations between water molecules. ${ }^{39}$ As illustrated in Fig. $1 \mathrm{C}$ the coherent contribution from ions is expected to vanish according to symmetry considerations: for every ion, other ions are on average distributed symmetrically around it. The vector sum of the electrostatic field from other ions at the location of a given central ion is on average zero $\left(\sum \boldsymbol{E}_{\text {ion-ion }}=0\right)$. The third-order contribution from individual ions therefore vanishes $\left(\boldsymbol{\beta}_{\text {cation/anion }}^{(3)} \sum \boldsymbol{E}_{\text {ion-ion }}=0\right)$. In addition, $\boldsymbol{\beta}_{\text {cation/anion }}^{(2)}=0$ for centrosymmetric ions. Therefore, the coherent response of electrolyte solutions originates from correlated asymmetric water molecules, for which $\boldsymbol{\beta}_{\mathrm{H}_{2} \mathrm{O}}^{(2)} \neq 0$ and $\boldsymbol{\beta}_{\mathrm{H}_{2} \mathrm{O}}^{(3)} \cdot \boldsymbol{E}_{\mathrm{ion}-\mathrm{H}_{2} \mathrm{O}}(0) \neq 0$. Structural correlations or orientational order is represented by the second term in eqn (2). Ion-induced long-range changes in the orientational order of water molecules were recently observed in aqueous electrolyte solutions. ${ }^{39}$ This orientational order gives rise to an ion-induced coherent $\mathrm{SH}$ water signal in only two (PPP, PSS) of the four accessible polarization states (with collinear illumination). The orientational order that is probed in this way reports for a significant part on the breaking and formation of H-bonds. ${ }^{39}$ As illustrated in the inset of Fig. 1C, the motion of water molecules can be thought of as arising from bending and stretching motions of the H-bond. Coherent fs-ESHS probes orientational correlations and is therefore sensitive to bending of the $\mathrm{H}$-bond, but not to stretching of the $\mathrm{H}$-bond for which the orientational correlation between two water molecules does not change.

The orientational order of water can be qualitatively (not quantitatively) described by a DH model. ${ }^{39}$ The coherent fs-ESHS intensity is found to be related to the structure factor $S(q, c)$ of a gas of water dipoles in contact with ions: ${ }^{39}$

$$
I_{\mathrm{coh}}(2 \omega)_{J} \propto c\left|P_{\mathrm{i}-\mathrm{w}}^{(2)}(2 \omega)_{J}\right|^{2} S(q, c),
$$

where $P_{\mathrm{i}-\mathrm{w}}^{(2)}(2 \omega)_{J}$ is the second-order polarization induced in water by a single ion. $q$ is the scattering vector $\left(\boldsymbol{q} \equiv \boldsymbol{k}_{2 \omega}-2 \boldsymbol{k}_{\omega}\right)$. $S(q, c)$ reflects the relative positions of ions in an aqueous solution that is modeled as a uniform dielectric material. Utilizing the $\mathrm{DH}$ model to describe the molecular correlations in the electrolyte solution, $S(q, c)$ is given by

$$
\begin{aligned}
S(q, c) & =\frac{q^{2}}{q^{2}+K_{\mathrm{D}}(c)^{2}}, \\
\kappa_{\mathrm{D}}(c) & =\sqrt{\frac{\left(z_{\text {cation }}^{2}+j z_{\text {anion }}{ }^{2}\right) c N_{\mathrm{A}} e^{2}}{\varepsilon_{0} \varepsilon k_{\mathrm{B}} T}},
\end{aligned}
$$

where $\kappa_{\mathrm{D}}(c)$ is the DH screening parameter, $z_{\text {cation }}\left(z_{\text {anion }}\right)$ the valency of cations (anions), $N_{\mathrm{A}}$ Avogadro's number, $e$ the elementary charge, $\varepsilon_{0}$ the vacuum permittivity, $\varepsilon$ the static relative permittivity of water (at $293 \mathrm{~K}$ ), $k_{\mathrm{B}}$ the Boltzmann constant, and $T$ the temperature in $\mathrm{K}: j=1$ for chloride salts with monovalent cations and $j=2$ for divalent cations.

For the coherent PPP fs-ESHS intensity measured at a scattering angle $\theta$ of $90^{\circ}$ the orientational distribution of water dipoles can be computed in terms of ion-dipole interactions and thermal fluctuations. Using the linearized Boltzmann distribution we find the second-order polarization that is induced in the water molecules: ${ }^{39,59}$

$$
\begin{aligned}
& P_{\mathrm{i}-\mathrm{w}}^{(2)}(2 \omega)_{Z}=N_{\mathrm{m}}\left(\widetilde{\beta}_{Z X X}^{(3)}+\frac{\mu \widetilde{\beta}_{Z X X}^{(2)}}{3 k_{\mathrm{B}} T}\right) E(\omega)_{X}^{2} \int E\left(0 ; \boldsymbol{r}^{\prime}\right) \frac{\boldsymbol{r}^{\prime} \cdot \boldsymbol{e}_{Z}}{\left|\boldsymbol{r}^{\prime}\right|} \mathrm{e}^{i \boldsymbol{q}_{90} \cdot \boldsymbol{r}^{\prime}} \mathrm{d}^{3} \boldsymbol{r}^{\prime}, \\
& \tilde{\beta}_{Z X X}^{(2)}=\frac{1}{5} \sum_{a}\left(2 \beta_{z a a}^{(2)}-\beta_{a a z}^{(2)}\right), \widetilde{\beta}_{Z X X}^{(3)}=\frac{1}{15} \sum_{a, b}\left(\beta_{a b b a}^{(3)}+2 \beta_{a a b b}^{(3)}\right),
\end{aligned}
$$

\begin{tabular}{|c|c|c|c|c|c|c|c|c|c|c|}
\hline Cations & $\mathrm{Mg}^{2+}$ & $\mathrm{Ca}^{2+}$ & $\mathrm{Sr}^{2+}$ & $\mathrm{Li}^{+}$ & $\mathrm{Na}^{+}$ & $\mathrm{NH}_{4}{ }^{+}$ & $\mathrm{Rb}^{+}$ & $\mathrm{Cs}^{+}$ & $\mathrm{K}^{+}$ & $\mathrm{Cl}^{-}$ \\
\hline$R(\mathrm{~nm})$ & 0.065 & 0.099 & 0.116 & 0.068 & 0.095 & 0.148 & 0.149 & 0.169 & 0.133 & 0.181 \\
\hline
\end{tabular}

where $N_{\mathrm{m}}$ is the number density of water molecules, $\mu$ the permanent dipole moment of a water molecule, and $\boldsymbol{e}_{Z}$ the unit vector in the $Z$ direction which contributes to $P$ polarized $\mathrm{SH}$ light, and $\left|\boldsymbol{q}_{90}\right|=\sqrt{2}\left|\boldsymbol{k}_{0}\right|$. With $E\left(0 ; \boldsymbol{r}^{\prime}\right)$ of a single ion given by the Coulomb equation, the integral $\int E\left(0 ; \boldsymbol{r}^{\prime}\right) \frac{\boldsymbol{r}^{\prime} \cdot \boldsymbol{e}_{Z}}{\left|\boldsymbol{r}^{\prime}\right|} \mathrm{e}^{i q_{90} \cdot \boldsymbol{r}^{\prime}} \mathrm{d}^{3} \boldsymbol{r}^{\prime}=\frac{Z e}{q} \frac{f(0)}{\varepsilon_{0} \varepsilon}$, in which

Table 1 Effective ionic radii, ${ }^{56,57}$ used for the calculation of the incoherent $\mathrm{SH}$ intensity from individual ions (Fig. $2 \mathrm{~A}$ ) 
$f(0)$ is the local field factor for the ionic electrostatic field. Note that the linearization of the Boltzmann distribution to obtain $\frac{\mu \widetilde{\beta}_{Z X X}^{(2)}}{3 k_{\mathrm{B}} T}$ in eqn (9) is typically valid for ion-dipole distances exceeding $1 \mathrm{~nm}$, i.e. $\left|\boldsymbol{r}^{\prime}\right| \geq 1 \mathrm{~nm}$, so that the single ion single dipole interaction energy is much weaker than the thermal energy $k_{\mathrm{B}} T$. As mentioned, in this calculation, water molecules are approximated as an ideal gas, for which water-water interactions are neglected. Using eqn (7)-(9), we compute the coherent $\mathrm{SH}$ response of water molecules in an electrolyte solution arising from the orientational correlations between water molecules induced by the total electrostatic field in solution originating from the ions. When there are no ions, there are thus no correlations between the water molecules.

Computational results. Fig. 2A shows the calculated incoherent $\mathrm{SH}$ intensity $I_{\text {ion }}(2 \omega)$ from individual ions in aqueous solutions of chloride salts with different cations. It can be seen that according to the computation (eqn (5) and (6)) $I_{\text {ion }}(2 \omega)$ increases linearly with the electrolyte concentration. There is no distinguishable difference in $I_{\text {ion }}$ for different monovalent cations and different divalent cations. The absence of ion specificity in $I_{\text {ion }}(2 \omega)$ is probably caused by the fact that the intensity scales with $R^{14}\left(\left|\overline{\boldsymbol{\beta}}_{\text {ion }}^{(3)}\right| \propto R^{7}\right.$, eqn (5)) and that the $\mathrm{Cl}^{-}$ ion $(R=0.181 \mathrm{~nm})$ is much bigger in size than any of the studied cations $(0.065 \mathrm{~nm}<R<0.169 \mathrm{~nm}$, see Table 1). In addition, $\left|\boldsymbol{E}_{\text {anion }}(0)\right|$ is more than twice larger than $\left|\boldsymbol{E}_{\text {cation }}(0)\right|$. The contribution from cations to $I_{\text {ion }}(2 \omega)$ is thus negligible. The difference between the divalent and monovalent series is caused by the different number of $\mathrm{Cl}^{-}$ions in solution; when the curves are corrected for the number difference in $\mathrm{Cl}^{-}$ions this difference vanishes.

The coherent concentration dependent response for bulk water is shown in Fig. 2B. The intensity increases at a very low electrolyte concentration. As discussed previously, ${ }^{39}$ this increase is caused by the combined electric field of all the ions inducing orientational correlations between the water molecules (see also ref. 43). As was noted in ref. 39, the actual increase in orientational order is 6 times smaller and displays an isotope effect of a factor of $\sim 3$. The reported difference in ref. 39 between the experimental data (which is also shown here in
Fig. 3C but with a linear concentration scale) and the theoretical $\mathrm{DH}$ curve in the concentration range from $1 \mu \mathrm{M}$ to $100 \mathrm{mM}$ is probably caused by the fact that the $\mathrm{DH}$ model neglects $\mathrm{H}$-bonding. For the present study we note that the $\mathrm{DH}$ model does not include any ion specificity, and the $\mathrm{SH}$ intensity remains constant up to $4 \mathrm{M}$.

\section{B. Experiments: fs-ESHS from electrolyte solutions}

The fs-ESHS intensities $\left(I_{\text {salt }}(2 \omega)\right)$ from aqueous electrolyte solutions were measured in SSS (Fig. 3A and B) and PPP (Fig. 3C) polarization combinations at a scattering angle of $\theta=90^{\circ}$ for electrolyte concentrations up to $4 \mathrm{M}$ (or approaching solubility limits for $\mathrm{SrCl}_{2}, \mathrm{RbCl}$, and $\mathrm{KCl}$ ). Fig. 3A and B present only incoherent responses and Fig. 3C presents both coherent and incoherent responses. The values for $I_{\text {salt }}(2 \omega)$ are normalized by that of pure water $\left(I_{\text {water }}(2 \omega)\right)$ measured in the same polarization combination. In both cases the curves were fit with a linear function of the form:

$$
I_{\text {salt }}(2 \omega) / I_{\text {water }}(2 \omega)=k_{\mathrm{PPP}(\mathrm{sss})^{\cdot}} \cdot c+b,
$$

where $k_{\mathrm{PPP}(\mathrm{SSS})}$ is the linear increase rate of the PPP (SSS) $I_{\text {salt }}(2 \omega) / I_{\text {water }}(2 \omega)$ ratio and $b$ is the interception of the $y$-axis. $k_{\mathrm{PPP}(\mathrm{Sss})}$ thus reflects the increase in the relative $\mathrm{SH}$ response if one molar of ions is added to the solution.

As shown in Fig. $3 \mathrm{~A}$ and $\mathrm{B}$, the measured incoherent response differs from ion to ion, and $k_{\mathrm{SSs}}$ changes from negative $\left(\mathrm{K}^{+}\right)$to almost zero $\left(\mathrm{Cs}^{+}\right)$and to positive (other cations). The linear increase is observed to start at $c>0.8 \mathrm{M}\left(\mathrm{Mg}^{2+}\right)$ or higher concentrations $\left(c>2 \mathrm{M}, \mathrm{Na}^{+}\right.$and $\left.\mathrm{Li}^{+}\right)$. The obtained fit values are given in Table 2. Comparing Fig. 2A to Fig. 3A and B, we observe differences between the computation and the measurement: the presence of ion specificity is not predicted by the theory nor is the large onset concentration of the intensity increase in Fig. 3A and B. Instead of incoherent single ion contributions that mainly arise from $\mathrm{Cl}^{-}$anions there must be another effect that dominates the SSS fs-ESHS intensity. As we have seen in eqn (2) the incoherent response of water is another source of fs-ESHS intensity. This response, which depends on the electronic structure of water, can possibly be influenced by ions in highly concentrated electrolyte solutions. ${ }^{28,29,60,61}$
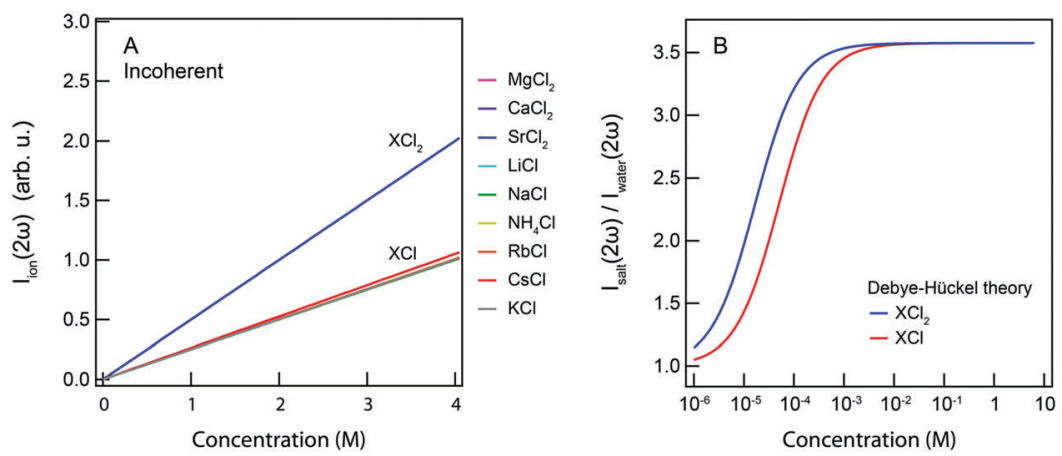

Fig. 2 Computed SH intensities from electrolyte solutions. (A) The incoherent SH intensities from individual ions are calculated based on eqn (5) and (6) for a cationic series of chloride salts. All monovalent and divalent cations behave approximately in the same way so that the curves overlap. (B) The coherent SH intensities of electrolyte solutions are calculated based on the DH theory (eqn (5)-(7)) for monovalent and divalent cations. There is no ion specificity in the calculated SH intensity. 

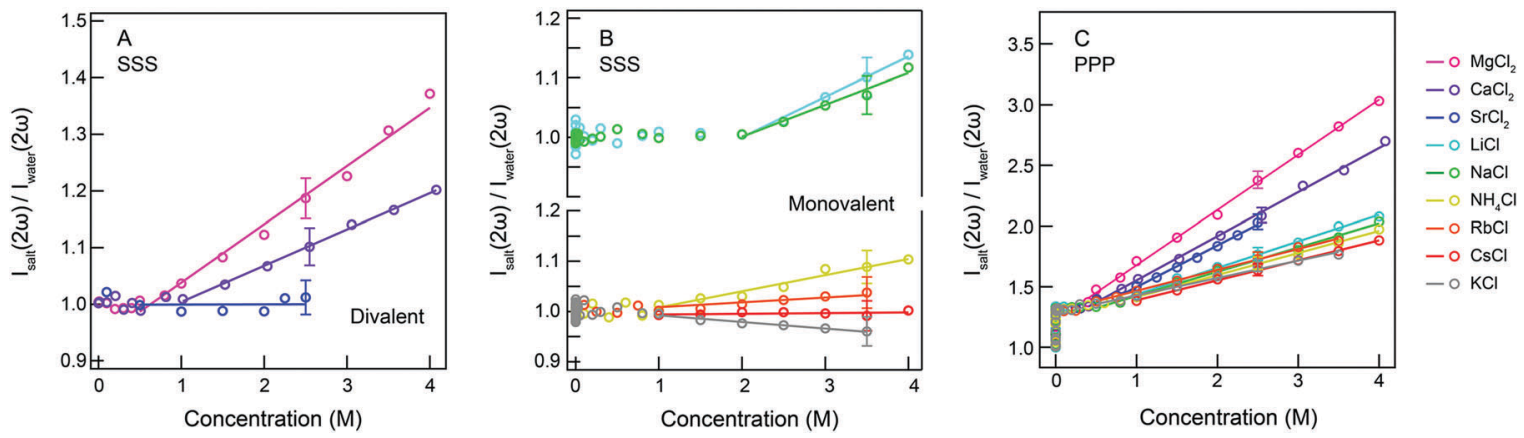

Fig. 3 Measured fs-ESHS intensities of electrolyte solutions. The fs-ESHS intensities $\left(I_{\text {salt }}(2 \omega)\right)$ of electrolyte solutions for the chloride salt series were measured in SSS (A and B) and PPP (C) polarization combinations. $I_{\text {salt }}(2 \omega)$ is normalized by the intensity of pure water $\left(I_{\text {water }}(2 \omega)\right)$ measured in the same polarization combination. The solid lines represent a linear fit of the fs-ESHS intensity. Note that the computed $y$-scale in Fig. 2A cannot be compared to the measured $y$-scale in Fig. 3.

Table 2 Fit parameters of the fs-ESHS data in Fig. 3 to eqn (10). Onset concentrations where the data start to increase linearly are also given

\begin{tabular}{llllllllll}
\hline Cation series & & $\mathrm{MgCl}_{2}$ & $\mathrm{CaCl}_{2}$ & $\mathrm{SrCl}_{2}$ & $\mathrm{LiCl}$ & $\mathrm{NaCl}$ & $\mathrm{NH}_{4} \mathrm{Cl}$ & $\mathrm{RbCl}$ & $\mathrm{CsCl}$ \\
\hline$k_{\mathrm{PPP}}\left(\mathrm{M}^{-1}\right)$ & & 0.45 & 0.36 & 0.34 & 0.22 & 0.20 & 0.18 & 0.17 & 0.16 \\
$k_{\text {SSS }}\left(\mathrm{M}^{-1}\right)$ & & 0.111 & 0.064 & 0.001 & 0.068 & 0.054 & 0.035 & 0.020 & 0.001 \\
Onset $(\mathrm{M})$ & PPP & 0.21 & 0.36 & 0.49 & 0.42 & 0.47 & 0.47 & 0.15 & 0.59 \\
& SSS & 0.79 & 0.94 & - & 2.00 & 1.99 & 0.75 & 1.12 & -0.013 \\
& & & & & & & -
\end{tabular}

The measured intensity changes start to appear at ion:water ratios of about $1: 14(\mathrm{NaCl})$ or $1: 23\left(\mathrm{MgCl}_{2}\right)$. At such concentrations ion-induced changes in the electronic structure of the hydration shells may be playing a role that could comprise charge transfer effects or ion pairing. Within this explanation, a decreasing intensity then implies that $\mathrm{K}^{+}$ions reduce the electronic anisotropy to a more spherical distribution of electron density, whereas an increase in intensity increases the amount of electronic anisotropy in the hydration shells. $\mathrm{Cs}^{+}$and $\mathrm{Sr}^{2+}$ do not or hardly influence the response of water, which means that the electronic structure of the closely associated water molecules is not different from that of bulk water. $\mathrm{MgCl}_{2}$ and $\mathrm{CaCl}_{2}$ do influence the electronic anisotropy in the neighboring water molecules, with $\mathrm{Mg}^{2+}$ having a bigger influence than $\mathrm{Ca}^{2+}$. This is expected because of the double valency and smaller size of $\mathrm{Mg}^{2+}$ compared to $\mathrm{Ca}^{2+}$. For the ions $\mathrm{Li}^{+}, \mathrm{Na}^{+}, \mathrm{NH}_{4}^{+}, \mathrm{Rb}^{+}$, and $\mathrm{Cs}^{+}$the amount of distortion is governed by the size of the ion. ${ }^{62,63}$ Why $\mathrm{K}^{+}$has an opposite effect on the electronic structure of water cannot be explained by the size and charge difference with respect to the other ions. In fact, in order to reduce the electronic anisotropy of the first hydration shell, the lone pair electron of the oxygen of water needs to shift towards the hydrogens of the same hydrating water molecule. The distribution of the lone pair electrons of the oxygen of water highly depends on the local environment, which can be changed by water H-bonding or the presence of ions, as indicated by previous oxygen K-edge X-ray emission spectroscopy measurements on liquid water. ${ }^{64}$

Turning now to the combined coherent and incoherent contributions (Fig. 3C), we observe that in the PPP polarization combination the fs-ESHS intensities show the same type of behavior: The fs-ESHS intensity increases linearly with electrolyte concentration. There are, however, two notable differences: Fig. 3C displays an increase of a larger magnitude (from 1.3 to 3 instead of from 1 to 1.3 for $\mathrm{MgCl}_{2}$ ) as well as an onset concentration that is up to a factor of 5 lower, bringing the ion: water ratios to hundreds of water molecules per ion. It is unlikely that at such low concentrations ions already induce a significant perturbation in the distribution of electronic charges of the hydration shell which is necessary to change $\boldsymbol{\beta}^{(2)} \cdot{ }^{17}$ For such low onset concentrations, and to generate a bigger change in the PPP fs-ESHS intensity, ion-induced structural correlations between water molecules are needed.

As reported recently, ${ }^{39}$ such ion-induced orientational correlations or orientational order are present in electrolyte solutions and can be observed with fs-ESHS in the PPP polarization combination for ionic strengths as low as $10 \mu \mathrm{M}$. These correlations occur as a weak response of the $\mathrm{H}$-bond network of water to the electric field of the ions and lead to an increasing relative fs-ESHS intensity up to $\sim 1.3$. The same response was observed for 21 different electrolytes. ${ }^{39}$ Fig. $3 \mathrm{C}$ shows that for increasing salt concentrations the normalized intensities start to diverge, which may be a consequence of the hydration shells becoming smaller and being influenced by the charge, electronic structure, and size of the ions. It can be seen in Fig. 3C that all the slopes are positive and that $k_{\mathrm{PPP}}$ follows the order: $\mathrm{K}^{+}<\mathrm{Cs}^{+}<\mathrm{Rb}^{+}<$ $\mathrm{NH}_{4}^{+}<\mathrm{Na}^{+}<\mathrm{Li}^{+}<\mathrm{Sr}^{2+}<\mathrm{Ca}^{2+}<\mathrm{Mg}^{2+}$, which resembles a direct cationic Hofmeister series (with $\mathrm{K}^{+}$and $\mathrm{NH}_{4}{ }^{+}$as exceptions). ${ }^{3,65}$ The exception of $\mathrm{NH}_{4}{ }^{+}$is likely due to its $\mathrm{H}$-bonding with water molecules, which induces changes in the orientational order of hydrating water molecules. A possible reason for the special behavior of $\mathrm{K}^{+}$might be that the water network becomes more disordered and the hydration shell becomes looser, as indicated by the larger ion-water oxygen 
distance than the oxygen-oxygen distance as well as the large ion-hydrogen distance measured by neutron diffraction. ${ }^{16,66}$ It can be seen that small divalent ions have the biggest influence on $k_{\mathrm{PPP}}$ and thus the most restricting influences on the rotational motion (or $\mathrm{H}$-bond bending mode) of water molecules. In contrast, larger monovalent ions have a smaller influence on restricting the $\mathrm{H}$-bond bending mode and lead to a lesser increase in the orientational order of water as represented by the PPP fs-ESHS intensity.

Fig. 2B shows the computed coherent relative $\mathrm{SH}$ intensity based on the DH theory. This curve reflects ion-induced orientational order in a dipolar gas of water molecules. This model does not describe the experimental data well. It can be seen that the $\mathrm{DH}$ model fails to capture the observed ion specificity. It does, however, show the extent over which ions could influence the orientational order of water if all water-water interactions would be turned off. As can be seen, at $4 \mathrm{M}$, the $\mathrm{MgCl}_{2}$ curve reaches a relative intensity of $I_{\text {salt }}(2 \omega) / I_{\text {water }}(2 \omega)=3$, which is close to the limiting value of the DH theory, namely $\sim 3.5$ (assuming the values for $\beta^{(2)}$ reported by Gubskaya and Kusalik $\left.{ }^{58}\right)$. This suggests that, at such high salt concentrations, the $\mathrm{Mg}^{2+}$-water interactions strongly contribute to the orientational order of water molecules. In previous X-ray Raman and small angle X-ray scattering studies by Nilsson and coworkers, ${ }^{18} \mathrm{Mg}^{2+}$ has also been shown to have strong interactions with water molecules and form tightly bound hydration shells.

As we have seen previously, ion-induced changes to the orientational order of water lead to changes in the free energy of the H-bond network of bulk water at electrolyte concentrations below $100 \mathrm{mM} .{ }^{39}$ We might expect similar changes, showing ion specificity, to occur as well in the macroscopic properties of bulk water that are sensitive to water-water interactions, at electrolyte concentrations higher than $100 \mathrm{mM}$. Two of the most well-known candidates are the viscosity of electrolyte solutions and the Gibbs free energy change of hydration of electrolytes, both bulk properties that display ion specificity. ${ }^{7,8,11,67,68}$

\section{Macroscopic properties of aqueous solutions of electrolytes}

Viscosity $\boldsymbol{B}$-coefficients. The resistance of a fluid to deformation by an external stress is described by the viscosity, which is a measure of intermolecular interactions, such as H-bonding in water. The dynamic viscosity of an aqueous electrolyte solution can be described by the empirical Jones-Dole equation ${ }^{7,67}$ as a power series of the electrolyte concentration $c$ in the solution:

$$
\eta / \eta_{0}=1+A c^{1 / 2}+B c,
$$

where $\eta$ is the dynamic viscosity of the solution, $\eta_{0}$ the viscosity of pure water, $A$ the viscosity $A$-coefficient, and $B$ the viscosity $B$-coefficient. The $A$-coefficient is thought to be related to ion-ion interactions and dominantly influences the value of $\eta / \eta_{0}$ for $c<0.05 \mathrm{M}$. The $B$-coefficient displays ion specificity, and is thought to reflect ion-water interactions influencing the value of $\eta / \eta_{0}$ for $c>0.05 \mathrm{M}^{7}$ In a traditional view, weakly hydrated ions with negative $B$-coefficients are referred to as "structure breakers" and strongly hydrated ions with positive $B$-coefficients are termed as "structure makers". 7,31,69 Even though this idea has been challenged, ${ }^{31}$ it is widely accepted that the $B$-coefficient is pertinent to the hydration of ions and ion-induced structural changes in the water network.

Ion-induced orientational order of water molecules can be understood as a restriction of the motion of water molecules. This restriction may influence the fluidity of the liquid and the motion of other objects in water and thus is related to changes in the viscosity. From Fig. 3C we observe that the onset electrolyte concentration of the linear increase of the PPP fs-ESHS intensity coincides with the concentration range where the $B$-coefficient starts to dominate (i.e. 0.05-0.1 M). As we have seen that the PPP fs-ESHS intensity is a measure of the ioninduced orientational order of water molecules, a correlation between the electrolyte-induced PPP fs-ESHS intensity increase and the viscosity $B$-coefficient may be expected. In Fig. $4 \mathrm{~A}$ we correlate $k_{\mathrm{PPP}}$ of the linear fit of the fs-ESHS intensity (Fig. 3C) with literature values ${ }^{7}$ of the viscosity $B$-coefficient for different electrolytes. The viscosity values are reproduced in Table 3 for completeness. The quality of the correlation is indicated by $R^{2}$, the coefficient of determination. A perfect correlation is achieved with $R^{2}=1 .^{48}$ It can be seen that there exists a very good correlation $\left(R^{2}=0.97\right)$ between the $B$-coefficient and $k_{\mathrm{PPP}}$. This correlation can be qualitatively explained by noting that viscosity is determined by the rate at which $\mathrm{H}$-bonds are broken and formed, which is what the fs-ESHS experiment probes (as illustrated in Fig. 1C).

In contrast, as can be seen in Fig. $4 \mathrm{~B}$, no clear correlation between $k_{\text {Sss }}$ and the viscosity $B$-coefficient can be observed $\left(R^{2}=0.41\right)$. The linear change of the SSS fs-ESHS intensity also starts at electrolyte concentrations much higher than $0.05 \mathrm{M}$ (e.g. $2 \mathrm{M}$ for $\mathrm{LiCl}$ ). Thus the electronic structure changes in the hydrating water molecules are not correlated with the $B$-coefficient. This is not entirely unexpected: viscosity is a property that depends more on intermolecular interactions than on changes in the molecular electronic structure. ${ }^{56}$

The viscosity $B$-coefficient of electrolyte solutions dominates the influence of ions on the structure of water for electrolyte concentrations $>0.05 \mathrm{M}^{7}$ A recent neutron diffraction study ${ }^{66}$ reasons that ion induced viscosity changes are not caused by changes in the H-bond network of water as the viscosity $B$-coefficient is correlated with the difference between the oxygen-oxygen distance in pure water and the ion-oxygen distance in an electrolyte solution. The experimental data supporting this argument are, however, incomplete since neutron diffraction is only sensitive to the first few hydration shells. Here, we observe a strong correlation between the viscosity $B$-coefficient and the ion-induced orientational order in water as measured by fs-ESHS, which reports on changes in hundreds of water molecules (i.e. the extended hydration shell). Qualitatively, this correlation can be understood since the ions pose a restriction to the degrees of freedom in the $\mathrm{H}$-bond network. As the resistance of water to objects moving through it is determined by the breaking and formation of $\mathrm{H}$-bonds it is clear that there can indeed be a connection. The viscosity of electrolyte solutions is thus highly related to the ion-induced distortions in the $\mathrm{H}$-bond network of water. 

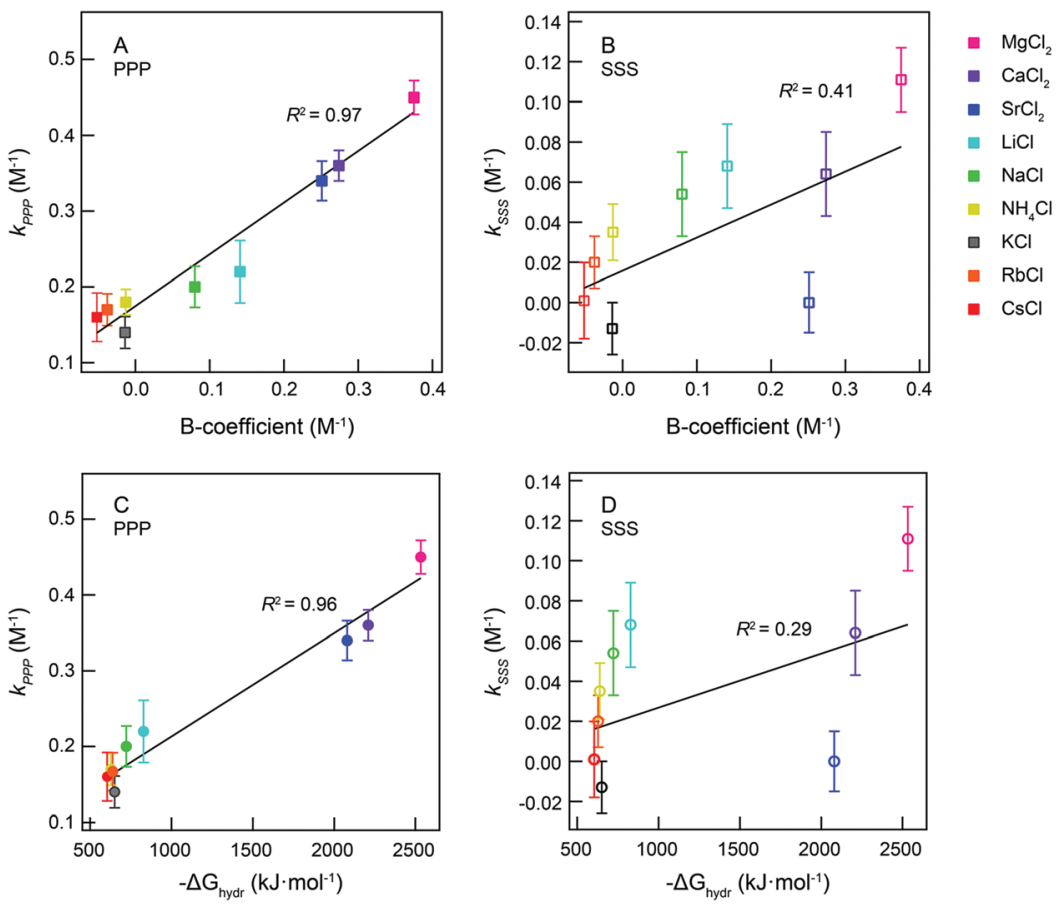

Fig. 4 Correlation between fs-ESHS increase and viscosity and Gibbs free hydration energy. (A) $k_{\mathrm{PpP}} v$ s. the viscosity $B$-coefficient for the monovalent and divalent cationic series of Fig. 3C. (B) $k_{\mathrm{Sss}} v s$. the viscosity $B$-coefficient for the monovalent and divalent cationic series in Fig. 3A and B. (C) $k_{\mathrm{PpP}} v s$. the Gibbs free energy change of hydration of electrolytes $\left(-\Delta G_{\text {hydr }}\right)$ for the monovalent and divalent cationic series of Fig. $3 C$. (D) $k_{\text {SSs }} v s$. $-\Delta G_{\text {hydr }}$ for the monovalent and divalent cationic series in Fig. 3A and B. The error bar in $k$ represents the $95 \%$ confidence interval of the linear fit of the fs-ESHS intensity to eqn (10). The values for the viscosity $B$-coefficient and hydration Gibbs free energy are taken from ref. 7 and 8 , respectively. They are reproduced in Table 3 .

Table 3 Viscosity $B$-coefficients ${ }^{7}$ and hydration free energy ${ }^{8}$ values of chloride salts as used in Fig. 4

\begin{tabular}{lllllllll}
\hline Cation series & $\mathrm{MgCl}_{2}$ & $\mathrm{CaCl}_{2}$ & $\mathrm{SrCl}_{2}$ & $\mathrm{LiCl}$ & $\mathrm{NaCl}$ & $\mathrm{NH}_{4} \mathrm{Cl}$ & $\mathrm{RbCl}$ & $\mathrm{CsCl}$ \\
\hline$B$-Coefficient $\left(\mathrm{M}^{-1}\right)$ & 0.375 & 0.274 & 0.251 & 0.141 & 0.080 & -0.013 & -0.038 & -0.052 \\
$-\Delta G_{\text {hydr }}\left(\mathrm{kJ} \mathrm{mol}^{-1}\right)$ & 2532 & 2209 & 2080 & 828 & 722 & 639 & 628 & 605 \\
\hline
\end{tabular}

Gibbs free energy change of electrolyte hydration. The Gibbs free energy change of hydration of electrolytes $\left(\Delta G_{\text {hydr }}\right)$ describes the total free energy change during the formation of hydrated individual ions (cations and anions) from pure liquid water and ions in the gas phase. The free energy change of water molecules in the ionic hydration shells dominates $\Delta G_{\text {hydr }}$, since $\Delta G_{\text {hydr }}$ is given in the limit of infinite dilution and thus ion-ion interactions are excluded. ${ }^{11}$ Thus, $\Delta G_{\text {hydr }}$ directly reflects the ion-induced energy changes in the extended hydration shells of ions.

In Fig. $4 \mathrm{C}$ and $\mathrm{D}$, we correlate the rate of change in the fs-ESHS intensity (Fig. 3) $-k_{\mathrm{PPP}}$ and $k_{\mathrm{SSS}}-$ with literature values ${ }^{8}$ of $-\Delta G_{\text {hydr }}$, respectively. As Fig. $4 \mathrm{C}$ shows, a strong correlation exists between $\Delta G_{\mathrm{hydr}}$ and $k_{\mathrm{PPP}}$ for the cation series of chloride salts, with $R^{2}=0.96$. Cations, that induce larger orientational order in the water network, characterized by a larger $k_{\mathrm{PPP}}$, are correlated with a larger value of $-\Delta G_{\text {hydr }}$. This means that ions, which constrain more water molecules, generate a bigger (negative) increase in the free energy of hydration. $\mathrm{Mg}^{2+}$ interacts with more water molecules than $\mathrm{Cs}^{+}$, which is energetically more favorable. As such, smaller ions with larger valencies induce larger structural changes in the water network that lead to a larger reduction in the Gibbs free energy of the water network for the ion hydration. $\Delta G_{\text {hydr }}$ is thus highly related to the ion-induced distortions in the $\mathrm{H}$-bond network of water. In contrast, there is no clear correlation $\left(R^{2}=0.29\right)$ between $k_{\mathrm{Sss}}$ and $-\Delta G_{\text {hydr }}$, as shown in Fig. $4 \mathrm{D}$. The ion-induced electronic changes, in the hydrating water molecules, are not correlated with the change in the total free energy of the solution. We can explain the correlation by noting that, $\Delta G_{\text {hydr }}$ mainly results from the ion-water and water-water interactions related to the orientational order of water molecules.

Thus, we find that the viscosity $B$-coefficient, $-\Delta G_{\mathrm{hydr}}$, and $k_{\mathrm{PPP}}$ increase in magnitude following a direct cation Hofmeister series. $^{3,65}$ It is also evident that for these three observable changes in the H-bond network play an important role. Recent MD simulations performed by Thomas and Elcock ${ }^{32}$ show that the influence of ions on the relative number of $\mathrm{H}$-bonds per water molecule in electrolyte solutions is ion specific and follows a direct Hofmeister series. Connecting both aspects there is a qualitative explanation for the observed correlations. What the exact molecular mechanism is for the observed increase 
in the fs-ESHS response and what the exact mechanism is behind the correlation observed in Fig. 4 need to be investigated in more detail. In addition, more sophisticated atomistic simulations that calculate the electronic anisotropy as well as the induced orientational order will be useful. Then, with such models both the fs-ESHS response and the viscosity need to be computed with various degrees of precision (and length/time scales).

\section{Conclusions}

We have probed ion-induced electronic and structural changes in water, for a cation series of chloride salts using fs-ESHS. For both the incoherent and coherent responses specific ion effects are observed above concentrations of $\sim 1 \mathrm{M}$ and $\sim 0.1 \mathrm{M}$, respectively. Comparing the changes in the molecular electronic anisotropy (incoherent response), as measured with fs-ESHS in the SSS polarization combination to theoretical considerations, we conclude that the spherical cations themselves do not significantly contribute to the measured fs-ESHS response.

As described in the theoretical description, an ion specific SSS response is rather originating from ion-induced changes in the electronic structure of water molecules adjacent to the ion. Positive changes as much as $37 \%\left(\mathrm{Mg}^{2+}, 4 \mathrm{M}\right)$ and negative changes of $-4 \%\left(\mathrm{~K}^{+}, 3.5 \mathrm{M}\right)$ indicate the extent of the impact of the ions on the electronic structure of hydrating water molecules. The increased responses are stronger for smaller ions with larger valencies. The $\mathrm{K}^{+}$-induced reduction in the fs-ESHS response is, however, anomalous. It means that $\mathrm{K}^{+}$ reduces the overall electronic anisotropy of water, for example via a redistribution of charge in an $\mathrm{H}$-bonding event. The difference in hydration between $\mathrm{K}^{+}$and $\mathrm{Na}^{+}$is particularly interesting in light of the unusual sensitivity of ion channels to either one of the species, ${ }^{35,36}$ and our experimental data might prove to be useful in elucidating the molecular cause of the functional difference.

The coherent PPP fs-ESHS response reports mainly on ioninduced changes in the structural orientational order of water in the extended hydration shells of the ions. Specific effects are observed that follow a Hofmeister trend. Here smaller ions lead to a larger increase in the fs-ESHS response, and the increasing valency also results in higher fs-ESHS intensity. The measured trend shows how cations restrict the orientational freedom of water, which is more effectively achieved for smaller ions with larger charges. A comparison to Debye-Hückel theory, which predicts the $\mathrm{SH}$ response for ions interacting with a dipolar gas of uncorrelated water molecules, suggests that $\mathrm{Mg}^{2+}$-water interactions, at a $\mathrm{Mg}^{2+}$ concentration of $4 \mathrm{M}$, strongly contribute to the orientational order of water molecules.

That such a reduction in the orientational freedom of water molecules might also influence macroscopic properties of bulk water was tested by examining correlations of the probed change in orientational order with the viscosity $B$-coefficient and the Gibbs free energy change of hydration $\left(\Delta G_{\text {hydr }}\right)$. Both the $B$-coefficient and $\Delta G_{\text {hydr }}$ are correlated well with the linear increase in the PPP fs-ESHS response. These observations show a possible connection between these three observables. This is exciting because the first two observables report on ion-water interactions at a macroscopic scale and the last observable at a molecular scale. A connection between these properties with the aid of more advanced theory than what is used here would undoubtedly further our understanding of the hydration of ions. This connection would also make it possible to connect the molecular and macroscopic properties of water with atomistic details. Given the omnipresent role that ions and water play in physics, chemistry, biology, and medicine this study is relevant for a wide variety of phenomena. The observed specific cation effects in both the electronic structure as well as the orientational ordering of water molecules provide us with a handle to understand and verify models aimed at understanding specific ion effects in water at both the molecular and macroscopic levels.

\section{Conflicts of interest}

There are no conflicts to declare.

\section{Acknowledgements}

This work was supported by the Julia Jacobi Foundation, the Swiss National Science Foundation (grant number 200021_140472), and the European Research Council (grant number 616305).

\section{References}

1 P. Lo Nostro and B. W. Ninham, Chem. Rev., 2012, 112, 2286-2322.

2 K. Collins, Methods, 2004, 34, 300-311.

3 W. Kunz, Specific Ion Effects, World Scientific Publishing Company, 2010.

4 P. Jungwirth and D. J. Tobias, Chem. Rev., 2006, 106, 1259-1281.

5 V. S. J. Craig, Curr. Opin. Colloid Interface Sci., 2004, 9, 178-184.

6 H. I. Okur, J. Hladílková, K. B. Rembert, Y. Cho, J. Heyda, J. Dzubiella, P. S. Cremer and P. Jungwirth, J. Phys. Chem. B, 2017, 121, 1997-2014.

7 H. D. B. Jenkins and Y. Marcus, Chem. Rev., 1995, 95, 2695-2724. 8 Y. Marcus, Ion properties, Marcel Dekker, New York, 1997.

9 W. Kunz, P. Lo Nostro and B. W. Ninham, Curr. Opin. Colloid Interface Sci., 2004, 9, 1-18.

10 H. Ohtaki and T. Radnai, Chem. Rev., 1993, 93, 1157-1204. 11 P. Hünenberger and M. Reif, Single-Ion Solvation: Experimental and Theoretical Approaches to Elusive Thermodynamic Quantities, Royal Society of Chemistry, 2011.

12 H. J. Bakker, Chem. Rev., 2008, 108, 1456-1473.

13 R. Mancinelli, A. Botti, F. Bruni, M. A. Ricci and A. K. Soper, Phys. Chem. Chem. Phys., 2007, 9, 2959-2967.

14 I. Howell and G. W. Neilson, J. Phys.: Condens. Matter, 1996, 8, 4455-4463.

15 S. Bouazizi, S. Nasr, N. Jaîdane and M.-C. Bellissent-Funel, J. Phys. Chem. B, 2006, 110, 23515-23523. 
16 S. Ansell, A. C. Barnes, P. E. Mason, G. W. Neilson and S. Ramos, Biophys. Chem., 2006, 124, 171-179.

17 I. Waluyo, D. Nordlund, U. Bergmann, D. Schlesinger, L. G. M. Pettersson and A. Nilsson, J. Chem. Phys., 2014, 140, 244506.

18 I. Waluyo, C. Huang, D. Nordlund, U. Bergmann, T. M. Weiss, L. G. M. Pettersson and A. Nilsson, J. Chem. Phys., 2011, 134, 064513.

19 J. D. Smith, R. J. Saykally and P. L. Geissler, J. Am. Chem. Soc., 2007, 129, 13847-13856.

20 Q. Sun, Vib. Spectrosc., 2012, 62, 110-114.

21 K. J. Tielrooij, N. Garcia-Araez, M. Bonn and H. J. Bakker, Science, 2010, 328, 1006-1009.

22 I. A. Heisler and S. R. Meech, Science, 2010, 327, 857-860.

23 S. Funkner, G. Niehues, D. A. Schmidt, M. Heyden, G. Schwaab, K. M. Callahan, D. J. Tobias and M. Havenith, J. Am. Chem. Soc., 2012, 134, 1030-1035.

24 A. W. Omta, M. F. Kropman, S. Woutersen and H. J. Bakker, Science, 2003, 301, 347-349.

25 J. S. Prell, J. T. O’Brien and E. R. Williams, J. Am. Chem. Soc., 2011, 133, 4810-4818.

26 J. T. O'Brien and E. R. Williams, J. Am. Chem. Soc., 2012, 134, 10228-10236.

27 R. Buchner and G. Hefter, Phys. Chem. Chem. Phys., 2009, 11, 8984 .

28 N. Ottosson, G. Öhrwall and O. Björneholm, Chem. Phys. Lett., 2012, 543, 1-11.

29 N. Ottosson, M. Odelius, D. Spångberg, W. Pokapanich, M. Svanqvist, G. Öhrwall, B. Winter and O. Björneholm, J. Am. Chem. Soc., 2011, 133, 13489-13495.

30 G. Stirnemann, E. Wernersson, P. Jungwirth and D. Laage, J. Am. Chem. Soc., 2013, 135, 11824-11831.

31 Y. Marcus, Chem. Rev., 2009, 109, 1346-1370.

32 A. S. Thomas and A. H. Elcock, J. Am. Chem. Soc., 2007, 129, 14887-14898.

33 M. Carrillo-Tripp, H. Saint-Martin and I. n. Ortega-Blake, J. Chem. Phys., 2003, 118, 7062.

34 Y. J. Zhang and P. S. Cremer, Annu. Rev. Phys. Chem., 2010, 61, 63-83.

35 Y. Zhou, J. H. Morais-Cabral, A. Kaufman and R. MacKinnon, Nature, 2001, 414, 43-48.

36 S. Varma and S. B. Rempe, Biophys. J., 2007, 93, 1093-1099.

37 K. D. Collins, Biophys. Chem., 2006, 119, 271-281.

38 S. J. Irudayam and R. H. Henchman, J. Chem. Phys., 2012, 137, 034508.

39 Y. Chen, H. I. Okur, N. Gomopoulos, C. Macias-Romero, P. S. Cremer, P. B. Petersen, G. Tocci, D. M. Wilkins, C. Liang, M. Ceriotti and S. Roke, Sci. Adv., 2016, 2, e1501891.

40 U. Baul, J. M. P. Kanth, R. Anishetty and S. Vemparala, J. Chem. Phys., 2016, 144, 104502.

41 A. P. Gaiduk and G. Galli, J. Phys. Chem. Lett., 2017, 8, 1496-1502.
42 H. I. Okur, Y. Chen, D. M. Wilkins and S. Roke, Chem. Phys. Lett., 2017, 684, 433-442.

43 D. M. Wilkins, D. E. Manolopoulos, S. Roke and M. Ceriotti, J. Chem. Phys., 2017, 146, 181103.

44 R. Bersohn, Y. H. Pao and H. L. Frisch, J. Chem. Phys., 1966, 45, 3184.

45 S. Roke and G. Gonella, Annu. Rev. Phys. Chem., 2012, 63, 353-378.

46 N. Gomopoulos, C. Lutgebaucks, Q. Sun, C. Macias-Romero and S. Roke, Opt. Express, 2013, 21, 815-821.

47 G. H. Golub and C. F. Van Loan, Matrix Computations, Johns Hopkins University Press, 3rd edn, 1996.

48 N. R. Draper and H. Smith, Applied Regression Analysis, Wiley, 1998.

49 J. W. Ponder, TINKER: Software Tools for Molecular Design, version 7.0, accessed May 20, 2017, http://dasher.wustl.edu/ tinker/.

50 M. L. Laury, L.-P. Wang, V. S. Pande, T. Head-Gordon and J. W. Ponder, J. Phys. Chem. B, 2015, 119, 9423-9437.

51 A. Grossfield, P. Ren and J. W. Ponder, J. Am. Chem. Soc., 2003, 125, 15671-15682.

52 T. Darden, D. York and L. Pedersen, J. Chem. Phys., 1993, 98, 10089.

53 G. J. Martyna, M. E. Tuckerman, D. J. Tobias and M. L. Klein, Mol. Phys., 1996, 87, 1117-1157.

54 R. W. Boyd, Nonlinear Opt., Academic Press, New York, 2008. 55 V. Kello, B. O. Roos and A. J. Sadlej, Theor. Chim. Acta, 1988, 74, 185-194.

56 P. Atkins and J. de Paula, Atkins Physical Chemistry, Oxford University Press, 2010.

57 J. N. Israelachvili, Intermolecular and Surface Forces, Academic Press, 2010.

58 A. V. Gubskaya and P. G. Kusalik, Mol. Phys., 2001, 99, 1107-1120.

59 D. P. Shelton and J. E. Rice, Chem. Rev., 1994, 94, 3-29.

60 D. Feller, E. D. Glendening, D. E. Woon and M. W. Feyereisen, J. Chem. Phys., 1995, 103, 3526.

61 M. D. Baer and C. J. Mundy, Faraday Discuss., 2013, 160, 89-101.

62 M. Dal Peraro, S. Raugei, P. Carloni and M. L. Klein, ChemPhysChem, 2005, 6, 1715-1718.

63 M. Soniat and S. W. Rick, J. Chem. Phys., 2012, 137, 044511. 64 T. Tokushima, Y. Harada, O. Takahashi, Y. Senba, H. Ohashi, L. G. M. Pettersson, A. Nilsson and S. Shin, Chem. Phys. Lett., 2008, 460, 387-400.

65 J. Traube, J. Phys. Chem., 1909, 14, 452-470.

66 T. Corridoni, R. Mancinelli, M. A. Ricci and F. Bruni, J. Phys. Chem. B, 2011, 115, 14008-14013.

67 G. Jones and M. Dole, J. Am. Chem. Soc., 1929, 51, 2950-2964.

68 W. M. Cox and J. H. Wolfenden, Proc. R. Soc. A, 1934, 145, 475-488.

69 K. D. Collins, Biophys. J., 1997, 72, 65-76. 